\title{
Agent-Based Modeling of an Air Quality Monitoring and Analysis System for Urban Regions
}

\author{
Mihaela Oprea \\ Petroleum-Gas University of Ploiesti, Department of Automation, \\ Computers and Electronic, \\ Bd. Bucuresti nr. 39, Ploiesti, 100680, Romania \\ mihaela@upg-ploiesti.ro
}

\begin{abstract}
Air quality is one of the main priorities for the improvement of the life quality in urban regions, as air pollution is usually, concentrated in such densely populated areas. Most of the countries have a national air quality monitoring network that allow an analysis of the air quality status, especially for urban regions that are nodes in this network. As the network is geographically distributed, it can be mapped in a natural way on an intelligent agents based system. The paper describes the modeling framework of an air quality monitoring and analysis multiagent system for urban regions.
\end{abstract}

\section{$1 \quad$ Introduction}

The quality of life in urban regions is related mainly to the indoor and outdoor environment quality, and one of the most important contribution is that of air quality (AQ) [22]. An efficient air quality management system can be viewed as a geographically distributed system mapped on an air quality monitoring network, that is modeled as an intelligent system based on agents. In the case of an environmental agent, a physical connection with sensors and/or measurement devices may exist, allowing the data acquisition from the environment (e.g. from the atmosphere), in certain sites from the environmental monitoring network. The agents can be equipped with various artificial intelligence techniques (e.g. data mining, case based reasoning, expert systems, fuzzy systems, artificial neural networks, swarm intelligence in the case of large environmental monitoring networks etc) in order to raise their performance in real time running by doing tasks specific to environmental management. Several research works reported the use of such techniques in environmental monitoring and analysis systems (see e.g. [1], [5], [6], [7], [9], [10], [11], [12], [14], [15]), not related directly with intelligent agents.

In this paper we propose an agent-based modeling framework for the development of urban air quality monitoring and analysis systems, where air pollution is much higher than in other regions. Despite the fact that there are some experimental agentbased environmental monitoring systems that were reported in the literature (see e.g. [2], [3], [4], [13]), there is no detailed formal description of the agent based model that was designed. Our framework provides a formal definition of such an agentbased model. 


\section{Air Pollution Monitoring and Analysis}

In the last decades, air quality became one of the main priorities for the improvement of the quality of life in urban regions (see e.g. [5], [8]), as air pollution is more concentrated in such densely populated areas. Most of the countries have a national air quality monitoring network, geographically distributed over the whole territory of the country, including as network nodes all or the most important urban regions sites, that allow the acquisition of the air pollutants concentrations based on in situ or remote sensing measurements. The networks provide an analysis of the air pollution status related to national and international air quality standards or regulations. For each urban region there are several sites geographically distributed over its territory, in the most important areas. Every site has specific air pollutants that are monitored, according to the major air pollution sources from the site neighbourhood (e.g. traffic pollution, chemical or petrochemical plants). Figure 1 shows the general view of a national air quality monitoring (AQM) network, composed by $n$ urban regions.

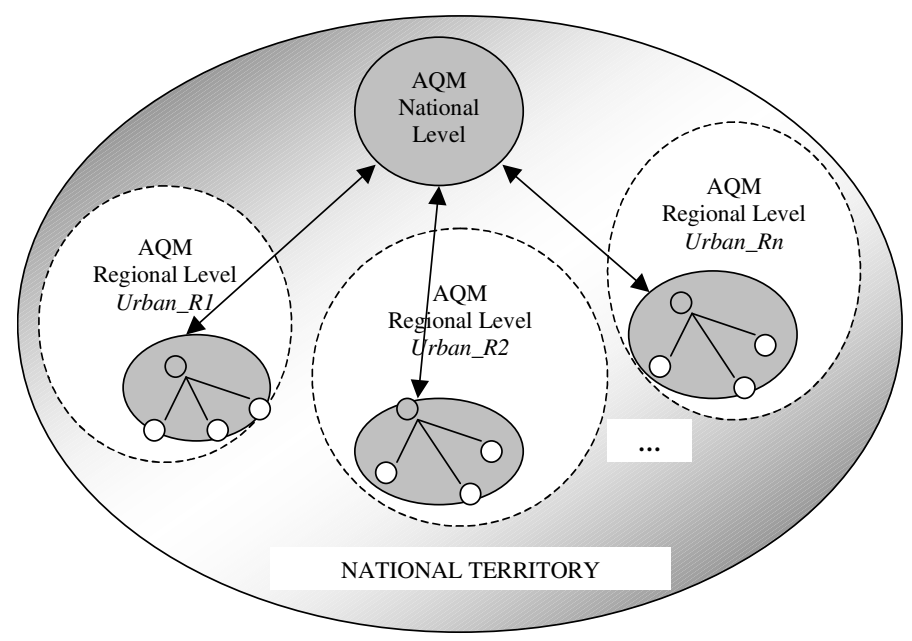

Fig. 1. The general view of a national air quality monitoring (AQM) network

The air quality analysis can be extended from the conformance with the national and international air quality standards to the estimation of air pollution impact on the human health (for sensitive categories of population such as children, elderly people, people with various health problems and ecosystems). The analyses are performed by using a variety of software instruments that are usually based on artificial intelligence techniques (traditional or computational intelligence techniques), and applied to the historical data stored in local or central databases or to the online data. The air pollution monitoring and analysis results are used by the decision factors of the environmental management system at regional or national level, in order to perform air quality control through air pollution reduction measures and to inform the population about the air quality status and its possible impact on human health. 


\section{The Multiagent Model of an AQ Monitoring System}

The national air quality monitoring network is composed by local air quality monitoring sites, specific to each urban regions that are included as nodes in this network. As the national AQM network is geographically distributed, it can be mapped in a natural way on an intelligent agents based system [16], i.e. each site has associated an agent (station agent), the local air quality supervision at the urban region level is done by an agent (urban region supervisor agent), and the national air quality supervision is done by another agent (national supervisor agent). The degree of air pollution is influenced by the meteorological factors, and thus, a meteo agent is included in the system in order to provide the meteo information. In an agent based modeling framework, an intelligent agent is an autonomous software entity that reacts to the changes in its environment, capable of social interactions (usually, by communication), and pro-active (guided by a goal and having initiative).

Figure 2 shows the architecture of the air quality monitoring and analysis multiagent system (AQMAS) that we propose for urban regions.

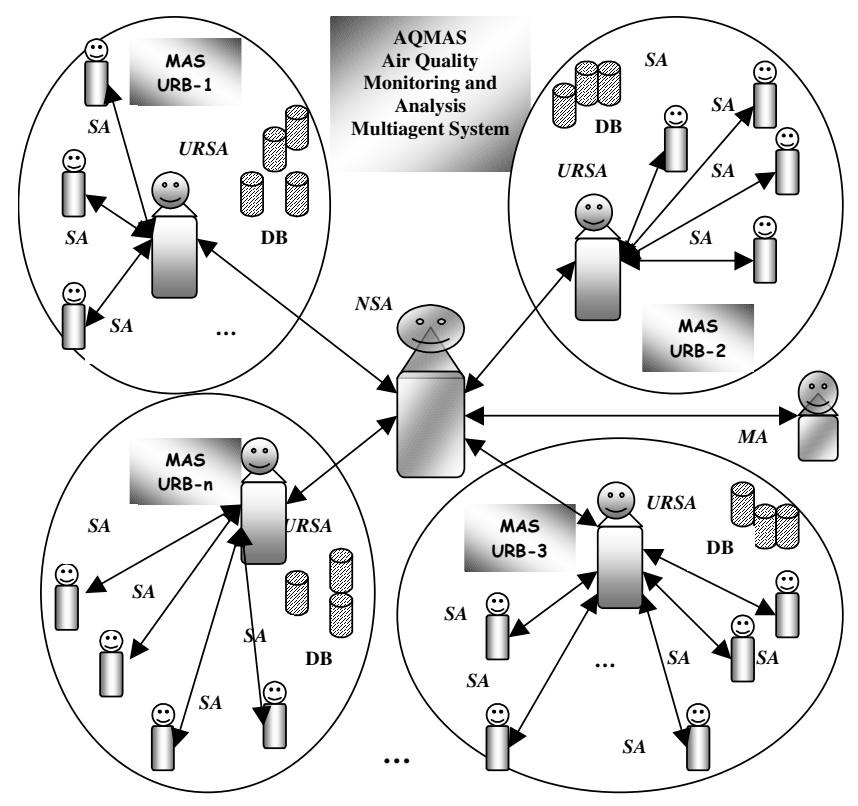

Fig. 2. The architecture of the Air Quality Monitoring and Analysis Multiagent System

The multiagent system has four types of agents: NSA (National Supervisor Agent), URSA (Urban Region Supervisor Agent), SA (Station Agent), MA (Meteo Agent). In our model, each urban region that has air quality monitoring sites included in the national AQM network has a local AQM network viewed as a smaller multiagent system (MAS-URB-i), composed by the agents associated to its local sites. 
The tasks performed by each agent are as follows:

$\begin{array}{ll}\text { NSA } \rightarrow \quad & \mathrm{T}_{1}-\text { NationalAQMonitoringAnalysis, } \\ & \mathrm{T}_{2}-\text { InformNationalAQReport, } \\ & \mathrm{T}_{3}-\text { RequestUrbanRegionAQReport, } \\ & \mathrm{T}_{4}-\text { RequestMeteoInformation, } \\ & \mathrm{T}_{5}-\text { NationalAQForecast; } \\ \text { URSA } \rightarrow \quad & \mathrm{T}_{1}-\text { UrbanRegionAQMonitoringAnalysis, } \\ & \mathrm{T}_{2}-\text { RequestStationAQReport, } \\ & \mathrm{T}_{3}-\text { RequestMeteoInformation, } \\ & \mathrm{T}_{4}-\text { SendUrbanRegionAQReport, } \\ & \mathrm{T}_{5}-\text { UrbanRegionAQForecast; } \\ & \mathrm{T}_{1}-\text { LocalAQMonitoringAnalysis, } \\ & \mathrm{T}_{2}-\text { MeasureAirPollutantConcentration, } \\ & \mathrm{T}_{3}-\text { CheckAirQualityStandard, } \\ & \mathrm{T}_{4}-\text { SendStationAQReport, } \\ & \mathrm{T}_{5}-\text { LocalAQForecast; } \\ & \mathrm{T}_{1}-\text { MeteoReport, } \\ & \mathrm{T}_{2}-\text { SendMeteoInformation; }\end{array}$

Some of the tasks are primitive, while others are composed tasks that can be decomposed in primitive tasks. For example, the task $\mathrm{T}_{1}$ performed by an SA agent LocalAQMonitoringAnalysis is a composed task that perform two primitive tasks: MeasureAirPollutantConcentration $\left(\mathrm{T}_{2}\right)$ and CheckAirQualityStandard $\left(\mathrm{T}_{3}\right)$. Some of the primitive tasks can involve just information exchange through messages. For example, the tasks $\mathrm{T}_{2}, \mathrm{~T}_{3}, \mathrm{~T}_{4}$ (for the NSA agent), $\mathrm{T}_{2}, \mathrm{~T}_{3}, \mathrm{~T}_{4}$ (for an URSA agent), $\mathrm{T}_{4}$ (for an SA agent) and $\mathrm{T}_{2}$ (for an MA agent). Other tasks, such as those performing air quality forecasting $\left(\mathrm{T}_{5}-\right.$ at local, urban region or national level) apply prediction methods (e.g. feed forward artificial neural networks, ARIMA).

The AQMAS model can be formally defined as a set given by relation (1).

$A Q M A S=\left\{N S A, M A, M A S-U R B-1, M A S-U R B-2, \ldots, M A S-U R B-n, M A S \_I n f r a s t r u c t u r e, A Q \_O n t o\right\}$

where, NSA is the national supervision agent that will centralize all information collected from local urban regions, MA is the meteo agent providing on request the meteorological forecasts and historical data, each urban region has its associated multiagent system (MAS-URB-i, defined as a set as given by relation (2)), $M A S \_I n f r a s t r u c t u r e$ includes the coordination and the communication mechanisms (i.e. protocols, interaction strategies etc), and the system resources (e.g. databases with air pollutants concentrations, air quality standards and regulations), AQ_Onto defines the ontology with terms specific to air quality monitoring and analysis.

$M A S-U R B-i=\left\{U R S A^{i}, S A_{l}, \ldots, S A_{j}, \ldots, S A_{m}, M A S-U R B-i \_I n f r a s t r u c t u r e, A Q \_O n t o\right\}$

where, each urban region has an urban region supervision agent $\left(U R S A^{i}\right)$, and a set of $m$ station agents $\left(S A_{j}\right)$ associated to the sites that monitor the air quality, the infrastructure of the urban region multiagent system (MAS-URB-i_Infrastructure) that 
includes the coordination and the communication mechanisms, and the system resources (e.g. databases with measurements of air pollutants concentrations, air quality standards and regulations, and the urban region information: region map with the location of the AQ monitoring sites and the air pollution sources with their location on the map, list of air pollutants specific to each site, list of possible human health effects, AQ color codes and associated warning messages for each air pollutant), and the air quality ontology (AQ_Onto). Each agent has specific resources provided by the databases included in the multiagent system infrastructure and a list of tasks to be performed.

Usually, an air quality analysis report can be asked to the NSA agent by the National Environmental Protection Agency (NEPA) or a governmental institution. Figure 3 presents an interaction diagram for such a scenario of interaction between the agents that compose the AQMAS system. The dashed rectangles represent the tasks executed by the agents. Thus, the task $\mathrm{T}_{1}$ is related to the specific agent that perform it (e.g. $\mathrm{T}_{1}$ performed by $\mathrm{SA}$ is LocalAQMonitoringAnalysis).

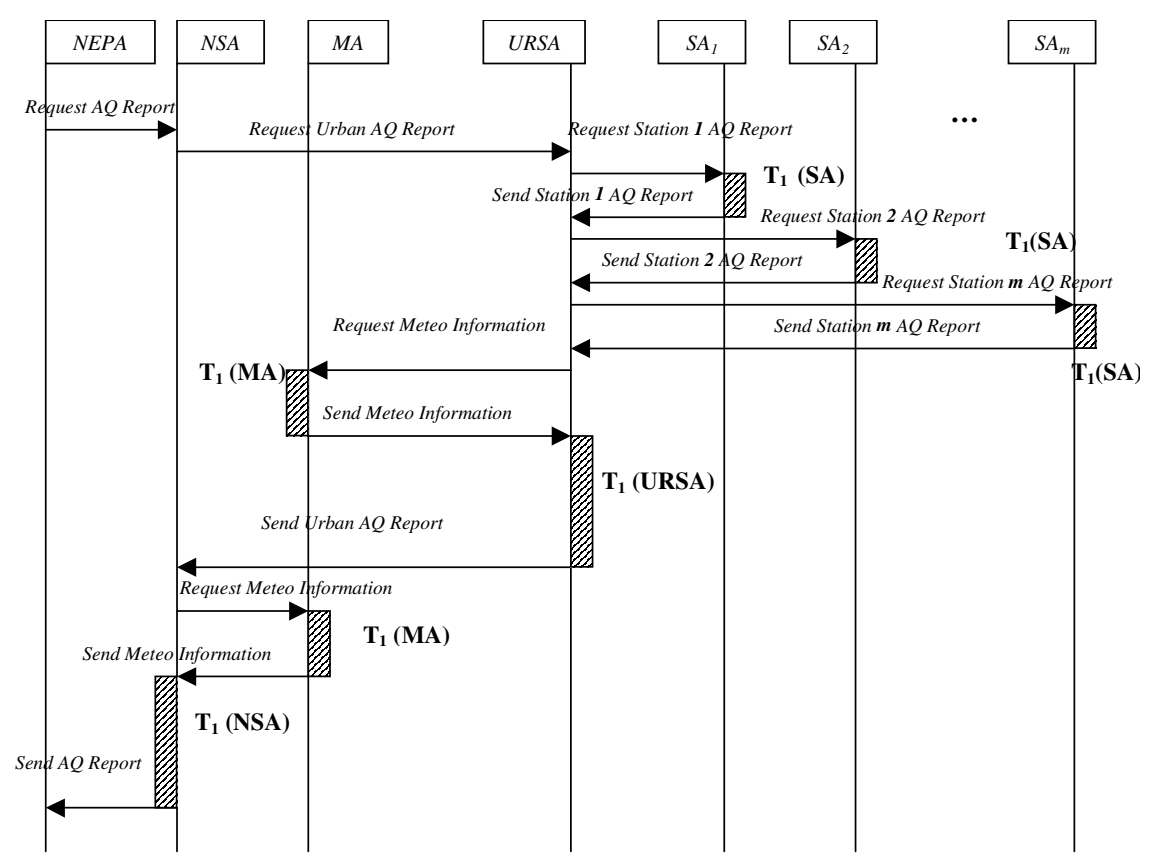

Fig. 3. Example of interactions diagram for a scenario of the AQMAS system run

The AQ_Onto ontology includes a conceptualization of the air quality monitoring and analysis domain, i.e. a vocabulary with all terms and concepts known by the agents involved in the AQMAS system run, as well as the relationships between these terms and concepts. The agents will solve domain specific problems via their abilities and information exchange through interagent comunication by using terms and concepts from the AQ_Onto vocabulary. Examples of terms and concepts used by the 
AQMAS system are as follows: AirPollutant, $C O, \quad P M, \quad S O 2$, AP_MaxAdmissibleConcentration, RegionMap, SiteLocation, AP_Concentration, WindSpeed, WindDirection, AirTemp, RelativeHumidity, AP_ColorCode, AP_ControlMeasure, AQI, AQ_Report. Each term and concept has a number of attributes with their domains of values, restrictions and default values. For example, the term AP_ColorCode has the attribute value of symbol type with values from the set $\{$ green, yellow, orange, red $\}$. The term $A Q I$ refers to the air quality index (see e.g. [18], [19]) that reports daily air quality and possible human health impact. AQI has a numerical value computed by taking into account the hourly concentrations of all air pollutants, measured at the site location, and specific intervals that allow the conversion from air pollutant concentration to an index, the maximum value being the returned index.

The proposed AQMAS system has an hierarchical architecture in which agents cooperate in order to solve the main goal of air quality monitoring and analysis for a cleaner air in urban regions.

\section{An Experimental AQ Monitoring and Analysis Multiagent System}

We have implemented a simplified version of the AQMAS system in Zeus [17], a Java based multiagent system development toolkit, for certain urban sites from the Romanian National Air Quality Monitoring Network [20]. Figure 4 shows the agents society of the experimental AQMAS system that was used in one of the simulations (three station agents - SA, one urban region supervision agent - URSA, the national supervision agent - NSA, and the meteo agent - MA).

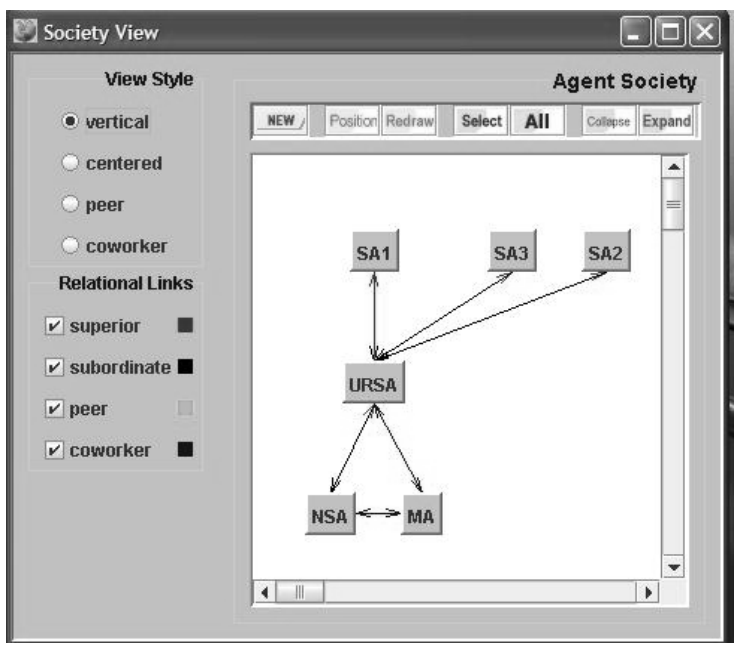

Fig. 4. The agent society of the AQMAS system for a simulation run 
The experimental system was tested as a simulation, the data (e.g. air pollutant concentrations and meteorological information) being taken from databases created with real data provided by the public sites [20] and [21].

Figure 5 shows a screenshot with the AQMAS project (aqmas.def) loaded in the Zeus Agent Generator 1.2.2. The ontology of the system (aqmas.ont) and the agents with their associated tasks can be easily created, viewed, and updated by using the corresponding editors (i.e. ontology editor, agent editor, task editor) as shown in the interface of the Zeus Agent Building Tool. The application source code generation is done automatically in Zeus. Another important advantage of using Zeus is the possibility of developing faster than other agent-based toolkits a prototype system for model validation.

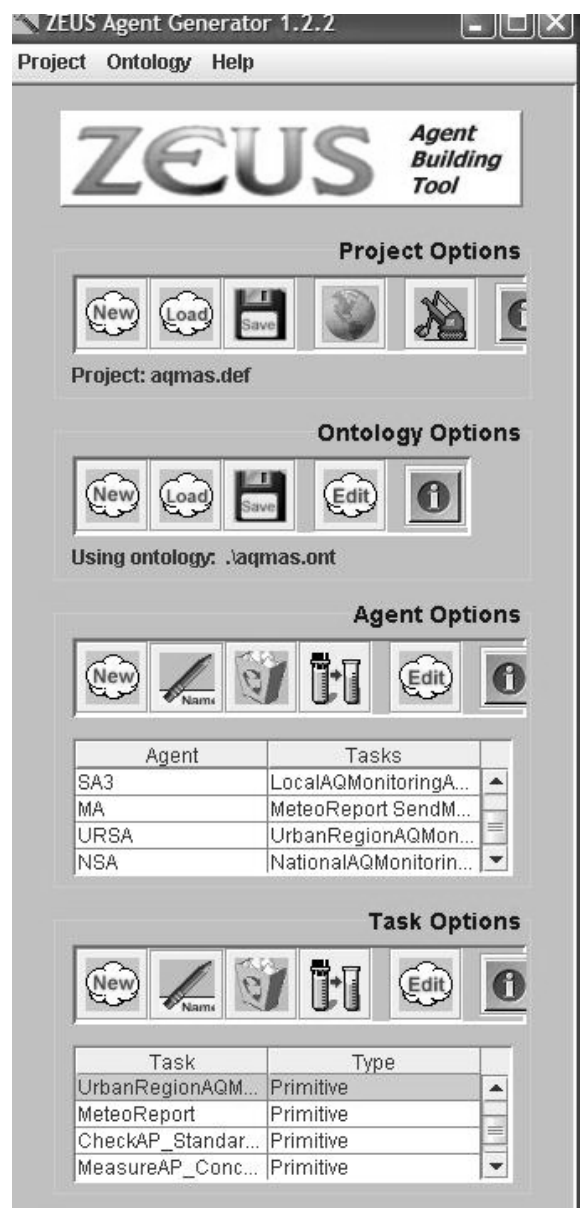

Fig. 5. Screenshot with the AQMAS system loaded in the Zeus Agent Generator 
Figure 6 presents a screenshot of the AQMAS system run for one urban region from the Romanian Air Quality Monitoring Network, the Ploiesti town, during May 2011. We have considered four sites, PH-1, PH-2, PH-3 and PH-4, distributed over the Ploiesti urban region, each site having a list of air pollutants that are monitored (i.e. hourly air pollutants concentrations measurements are recorded). A scenario similar with that presented in Figure 3 was run in order to validate the experimental AQMAS system. The URSA agent (assigned to the Ploiesti city) received the specific AQ standards checking reports from each local station agent $\left(\mathrm{SA}_{\mathrm{i}}\right.$ assigned to the sites $\mathrm{PH}-\mathrm{i}$ ) of the urban region network and provided to the national agent (NSA) the urban region AQ report. We can view in the central DOS window the AQ report provided by the $\mathrm{SA}_{1}$ agent assigned to the $\mathrm{PH}-1$ site, an yellow alert code for possible exceedance of the maximum admissible concentration of $\mathrm{CO}$ and $\mathrm{PM}_{10}$ air pollutants.

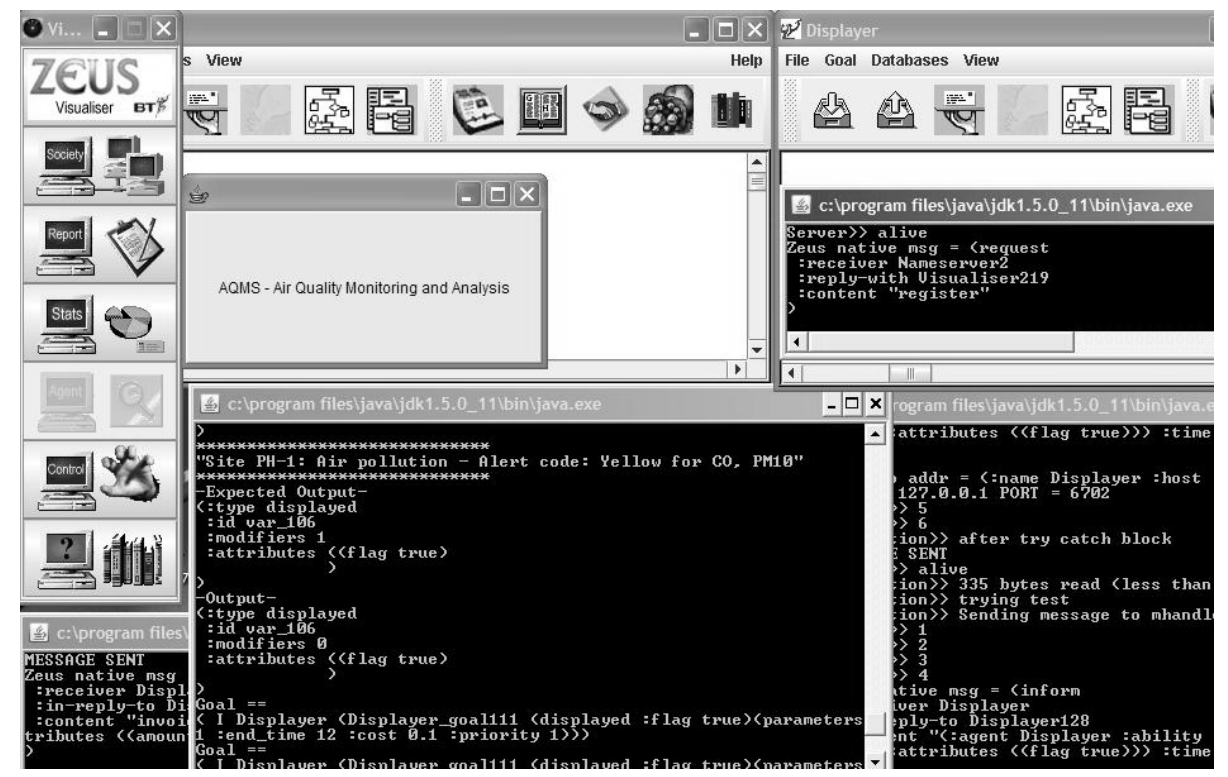

Fig. 6. Screenshot with the AQMAS system run for the Ploiesti urban region (May 2011)

\section{Conclusion and Future Work}

The paper presented a model of an agent-based air quality monitoring and analysis system, AQMAS, that was tested as a simulation for some urban regions from the Romanian National Air Quality Monitoring Network, providing alert codes when the maximum admissible limits of specific air pollutants concentrations were exceeded. As a future work we shall extend the functionality of the AQMAS experimental system with additional analysis capabilities including a human health air pollution impact analysis, an air pollution prediction and a multi-pollutant effects analysis. 


\section{References}

[1] Athanasiadis, I.N., Mitkas, P.A.: Knowledge Discovery for Operational Decision Support in Air Quality Management. Journal of Environmental Informatics 9(2), 100-107 (2007)

[2] Athanasiadis, I.N., Mitkas, P.A.: An agent-based intelligent environmental monitoring system. Management of Environmental Quality 15(3), 238-249 (2004)

[3] Di Lecce, V., Pasquale, C., Piuri, V.: A basic ontology for multi agent system communication in an environmental monitoring system. In: IEEE International Conference on Computational Intelligence for Measurement Systems and Applications (CIMSA), Boston, pp. 45-50 (2004)

[4] Kalapanidas, E., Avouris, N.: Air Quality Management Using a Multi-Agent System. Computer-Aided Civil and Infrastructure Engineering 17, 119-130 (2002)

[5] Karatzas, K.D.: Artificial Intelligence Applications in the Atmospheric Environment: Status and Future Trends. Environmental Engineering Management Journal 9(2), 171180 (2010)

[6] Kim, Y.J., Platt, U. (eds.): Advanced Environmental Monitoring. Springer (2008)

[7] Kolehmainen, M., Martikainen, H., Ruuskanen, J.: Neural networks and periodic components used in air quality forecasting. Atmospheric Environment 35, 815-825 (2001)

[8] Moussiopoulos, N. (ed.): Air Quality in Cities. Springer, Berlin (2003)

[9] Noori, R., Hoshyaripour, G., Ashrafi, K., Araabi, B.N.: Uncertainty analysis of developed ANN and ANFIS models in prediction of carbon monoxide daily concentration. Atmospheric Environment 44(4), 476-482 (2010)

[10] Núñez, H., Sànchez-Marrè, M., Cortés, U., Comas, J., Martinez, M., Rodríquez-Roda, I., Poch, M.: A comparative study on the use of similarity measures in case-based reasoning to improve the classsification of environmental system situations. Environmental Modelling \& Software 9(9), 809-819 (2004)

[11] Oprea, M.: INTELLEnvQ-Air: An Intelligent System for Air Quality Analysis in Urban Regions. International Journal of Artificial Intelligence 9(A12) (2012)

[12] Oprea, M.: A case study of knowledge modelling in an air pollution control decision support system. AiCommunications 18(4), 293-303 (2005)

[13] Oprea, M., Nichita, C.: On the Distributed Water Pollution Control Solving with an Agent-Based Approach. In: Badica, C., Paprzycki, M. (eds.) Advances in Intelligent and Distributed Computing. SCI, vol. 78, pp. 289-294. Springer, Heidelberg (2008)

[14] Polat, K.: A novel data preprocessing method to estimate the air pollution $\left(\mathrm{SO}_{2}\right)$ : neighbor-based feature scaling (NBFS). In: Neural Computing \& Applications. Springer (2011), doi:10.1007/s00521-011-0602-x

[15] Sànchez-Marrè, M., Gibert, K., Sevilla, B.: Evolving GESCONDA to an Intelligent Decision Support Tool. In: Proceedings of the International Congress on Environmental Modelling and Software Modelling for Environment's Sake, Fifth Biennial Meeting, Ottawa, Canada (2010)

[16] Weiss, G.: Multiagent Systems: A Modern Introduction to Distributed Artificial Intelligence. MIT Press, Cambridge (1999)

[17] Zeus Toolkit, http://www. labs.bt.com/projects/agents/zeus /

[18] http://airnow.gov

[19] http://www.airqualityontario.com

[20] http://www.calitateaer.ro

[21] http://www.meteoromania.ro

[22] http://www. who.int/ 